\title{
NEW PULSED ORBIT BUMP MAGNETS FOR THE FERMILAB BOOSTER SYNCHROTRON*
}

\author{
J.R. Lackey\#, J.A. Carson, C.M. Ginsburg, H.D. Glass, D.J. Harding, V.S. Kashikhin, A. Makarov, \\ E.J. Prebys \\ Fermi National Accelerator Laboratory, Batavia, IL 60510
}

\section{Abstract}

The beam from the Fermilab Linac is injected onto a bump in the closed orbit of the Booster Synchrotron where a carbon foil strips the electrons from the Linac's negative ion hydrogen beam. Although the Booster itself runs at $15 \mathrm{~Hz}$, heat dissipation in the orbit bump magnets has been one limitation to the fraction of the cycles that can be used for beam. New $0.28 \mathrm{~T}$ pulsed dipole magnets have been constructed that will fit into the same space as the old ones, run at the full repetition rate of the Booster, and provide a larger bump to allow a cleaner injection orbit. The new magnets use a ferrite in the yoke rather than laminated steel.

\section{INTRODUCTION}

The present injection system for the Fermilab Booster was designed in the early 1990s and was built with stacking operations to the Fermilab P-Bar Source in mind. Operation with repetition rates higher than $20 \%(3 \mathrm{~Hz})$ were never expected. Typical rates were on the order of $10 \%$. Currently the Booster provides protons for MiniBooNE and NUMI, as well as the P-Bar Source and will require the capability of repetition rates to $10 \mathrm{~Hz}$ or more. Due to severe heating in the present magnets the operational repetition rate is limited to $7.5 \mathrm{~Hz}$. New magnets have been designed which are capable of continuous operation at $15 \mathrm{~Hz}(100 \%$ duty factor $)$. These magnets have been designed to be radiation hard. The new magnets are stronger than the present magnets by $\sim 15 \%$. This increase in strength will allow injection into the Booster with the injection closed orbit at the center of the horizontal aperture rather than $1 \mathrm{~cm}$ radially outside of center as at present. This will significantly increase the horizontal injection aperture. These magnets will be installed in the Booster during the fall shutdown 2005.

In operation the magnets see a current waveform that rises roughly linearly in $20 \mu \mathrm{S}$ to a plateau at about $15 \mathrm{kA}$. The current is held constant during injection of multiple turns of beam, up to $35 \mu \mathrm{S}$. The current is then ramped down over $20 \mu \mathrm{S}$.

\section{CONSTRUCTION}

The magnet cross section is shown in Figure 1. The major differences between the new magnet design and the old are cooling for the conductor and the use of G4, a NiZn ferrite [1], for the magnet core instead of laminated

\footnotetext{
* Work supported by the United States Department of Energy under Contract No. DE-AC02-76CH03000

"lackey@fnal.gov
}

steel. The use of ferrite provides several benefits. Construction is simplified because the stack up of precision-machined ferrite blocks is much easier than stacking and gluing many thousands of very thin steel laminations. The amount of epoxy in the vacuum is reduced. It is also not necessary to insulate the current conductor or the ferrite core.

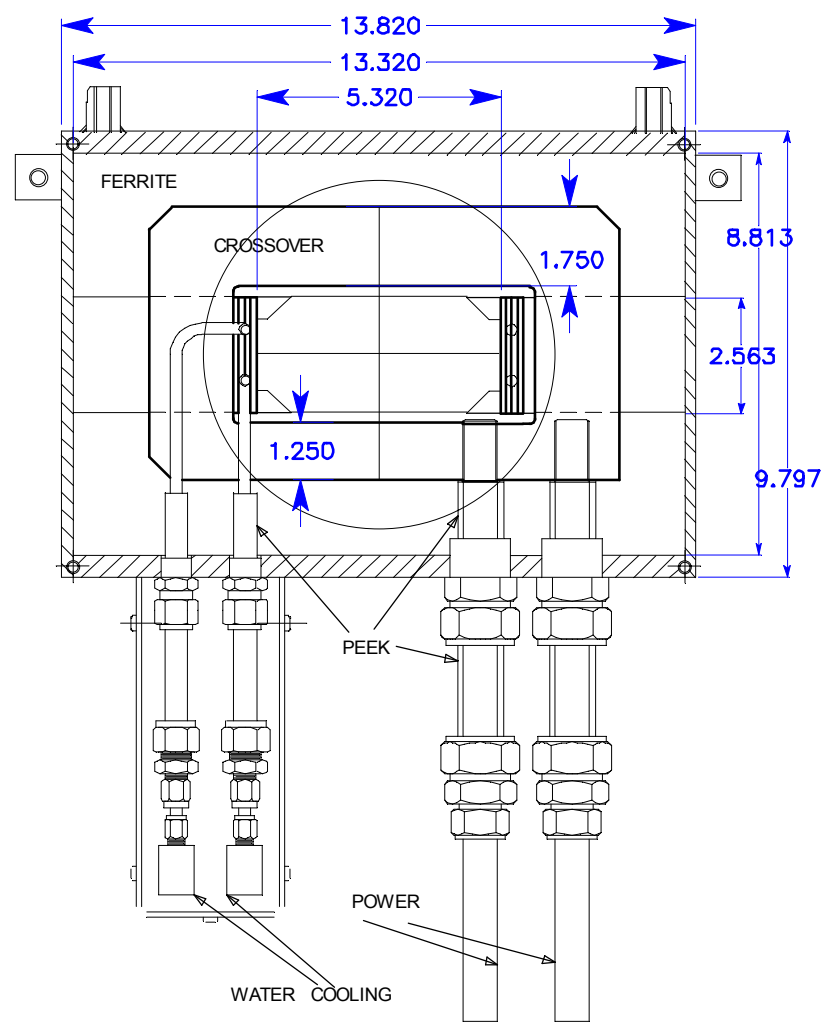

Figure 1 Magnet cross section and power and water leads. (Dimensions in inches)

Core power losses are considerably reduced and it is not necessary to actively cool the core. Any necessary core cooling is provided by direct conduction to the stainless steel skin of the magnet. It is estimated that the core loss is reduced from $\sim 1 \mathrm{~kW}$ to $\sim 200$ Watts by the use of ferrite instead of laminations.

The only electrical insulation used in the magnet is at the feed-through from vacuum to atmosphere for the power and cooling water. The insulating material is PEEK [2] which has excellent radiation resistant properties. Standard double ferrule fittings provide the vacuum seal between the copper current conductors, the PEEK insulators, and the stainless steel vacuum skin. The nominal magnet parameters are listed in Table 1. 
Table 1: Nominal Magnet Parameters

\begin{tabular}{|l|c|l|}
\hline Parameter & Value & Units \\
\hline JBy dl @ 15 kA & 0.1676 & $\mathrm{~T}-\mathrm{m}$ \\
\hline Ferrite length & 523.3 & $\mathrm{~mm}$ \\
\hline Effective length & 558.5 & $\mathrm{~mm}$ \\
\hline Aperture gap & 65.1 & $\mathrm{~mm}$ \\
\hline Aperture width & 135.1 & $\mathrm{~mm}$ \\
\hline Inductance & 1.83 & $\mu \mathrm{H}$ \\
\hline Resistance & $<1$ & $\mathrm{~m} \Omega$ \\
\hline
\end{tabular}

\section{FIELD CALCULATIONS}

During the magnet design the field was modeled in three dimensions at the operating frequency using Opera-3D [3]. There is a quadrupole component of the field at the lead end due to the asymmetrical configuration of the leads and their connection to the single turn coil. Figure 2 shows the main bending field $\mathrm{By}$ at $\mathrm{y}=0$ as a function $\mathrm{x}$ and $\mathrm{z}$.

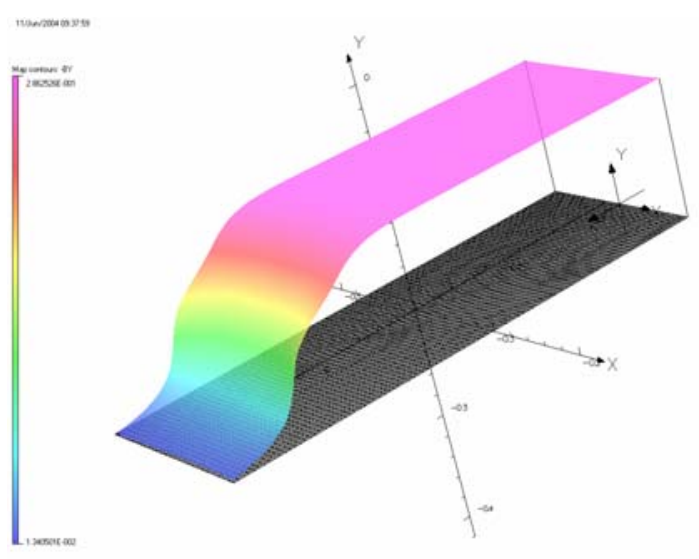

F VECTOR FIELDS

Figure 2 Calculated By at $\mathrm{y}=0$ as a function of $\mathrm{x}$ and $\mathrm{z}$ through the end of the magnet.

\section{FIELD MEASUREMENTS}

\section{Measurements}

Magnetic measurements have been made at a low DC current, $1700 \mathrm{~A}$, allowing precise determination of all field components due to geometrical effects. The primary measurement tool was a rotating tangential coil that extended through the magnet, measuring all components of the field integral. Figure 3 shows the integral of the main field, $\int \mathrm{By} \mathrm{dl}$, as a function of horizontal position, recreated from the harmonic components.
ORBD001, field shape from tangential coil

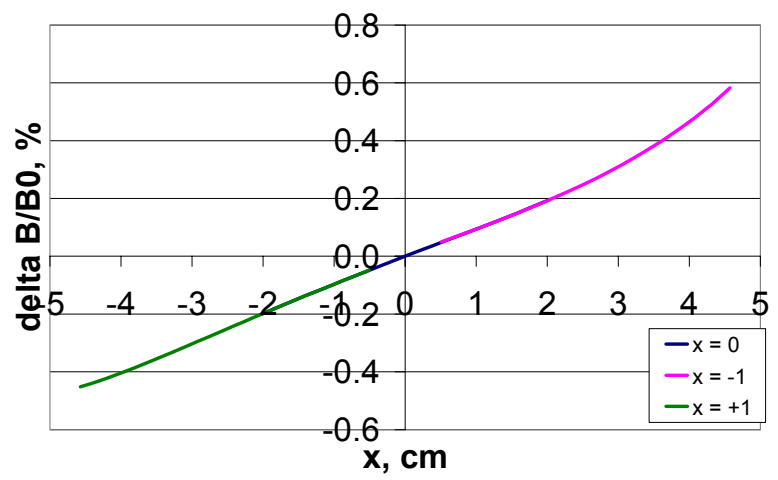

Figure 3 Fractional deviation from the central value of the $\int \mathbf{B y} \mathbf{d l}$ recreated from rotating coil measurements at three different $\mathrm{x}$ positions, $\mathrm{x}=0 \mathrm{~cm}, \mathrm{x}=-2.54 \mathrm{~cm}, \mathrm{x}=$ $+2.54 \mathrm{~cm}$.

\section{AC Measurements}

The power supply for the AC field measurement is a half-sine wave pulser that provides an output at the nominal peak current of $15 \mathrm{kA}$. It was not possible to excite the magnet with a flat-top pulse similar to the actual operational current for these measurements.

The measurements were taken using a long single turn Bdot coil so that the total field integral is measured. The probe is scanned from side to side across the aperture to provide field data as a function of position. The data are recorded, typically using a 20 pulse average, for both the Bdot coil and the power supply current simultaneously, with a $2.5 \mathrm{Msample} / \mathrm{sec}, 16$-bit digitizer. The Bdot signal is digitally integrated and the result is normalized to the nominal $15 \mathrm{kA}$ current. Table 2 and Figure 4 show the results of the field measurements on the first production magnet. Extrapolation of the DC field components from measurements at $500 \mathrm{~A}, 1000 \mathrm{~A}$, and $1700 \mathrm{~A}$ indicate that the field components measured at higher $\mathrm{AC}$ current are probably compatible with those measured with the 1700 A DC current. The measured DC components are listed in Table 2 also.

Table 2: Field Components @ 25.4 mm

\begin{tabular}{|c|c|c|}
\hline & $\begin{array}{c}\text { AC Measurements } \\
\mathbf{a} \text { 15 kAmps } \\
\mathbf{B}_{\mathbf{n}} / \mathbf{B}_{\mathbf{0}}\end{array}$ & $\begin{array}{c}\text { DC Measurements } \\
\text { @ } \mathbf{1 7 0 0} \text { Amps } \\
\mathbf{B}_{\mathbf{n}} / \mathbf{B}_{\mathbf{0}}\end{array}$ \\
\hline 0 & 1.0 & 1.0 \\
\hline 1 & $1.435 \mathrm{e}-3$ & $2.40 \mathrm{e}-3$ \\
\hline 2 & $4.782 \mathrm{e}-5$ & $-1.07 \mathrm{e}-5$ \\
\hline 3 & $2.173 \mathrm{e}-4$ & $1.27 \mathrm{e}-4$ \\
\hline 4 & $4.005 \mathrm{e}-5$ & $1.13 \mathrm{e}-5$ \\
\hline 5 & $-2.000 \mathrm{e}-5$ & $-1.54 \mathrm{e}-5$ \\
\hline 6 & $-2.297 \mathrm{e}-5$ & $-2.31 \mathrm{e}-5$ \\
\hline
\end{tabular}




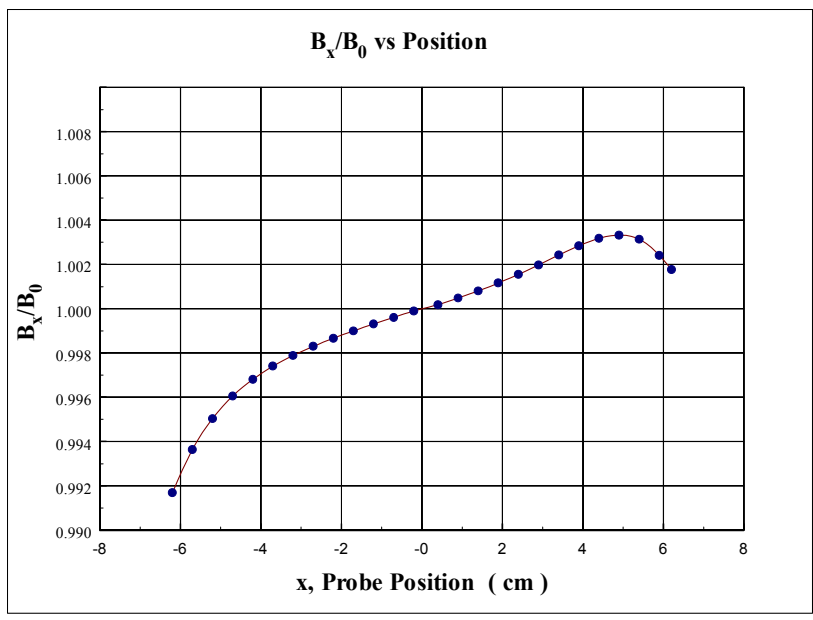

Figure 4 Field variation as a function of probe position. $\pm 1 \%$ full scale.

\section{DISCUSSION}

Magnet modeling prior to production had predicted some amount of quadrupole component due primarily to asymmetric current flow at the power feed end of the magnet and an attempt was made to compensate this effect by modifying the shape (see Figure 1) of the crossover conductors. A small quadrupole component is not a concern if the magnet is used in a four-magnet injection scheme similar to the present injection method, since the magnets can be arranged to cause dipole fold- down effects of the quadrupole components to cancel exactly. However, a new injection scheme [4] using only three bump magnets, has since been proposed and is going to be installed in the Booster. In this configuration the cancellation is not exact and will cause an orbit distortion of $\pm 2.5 \mathrm{~mm}$ while the bump magnet field ramps down at the end of the beam injection [5,6]. Various methods of compensation without rebuilding the magnet are being investigated. It is felt that this small orbit distortion will not be a significant problem even if it is left uncompensated.

\section{REFERENCES}

[1] G4, National Magnetic Group, Inc. 1210 Win Drive, Bethlehem, Pennsylvania 18017- 7061 USA

Also available elsewhere as CMD10

[2] PEEK $^{\mathrm{TM}}$ Victrex ${ }^{\circledR}$. Victrex plc, Victrex Technology Centre, Hillhouse International, Lancashire FY5 4QD

[3] Vector Fields Limited, 24 Bankside, Kidlington, Oxford, OX5 1JE, England

[4] M.B. Popovic, A Proposed H- Injection System for the Fermilab Booster, Beamdoc-1784, 2005.

[5] E.J. Prebys, 2005, FNAL unpublished document.

[6] A. Drozhdin, 2005, FNAL unpublished document. 